\title{
Reglas informales en los consejos de cuenca. El caso del río Santiago
}

\section{Informal rules in the basin councils. The case of the Santiago River}

doi: http://dx.doi.org/10.32870/

espiral.v26i74.7047

\section{Resumen}

En este artículo,se analiza la implementación de una política hídrica en el río Santiago (México), con énfasis en la participación social en la gestión del agua, resaltando el papel de las reglas informales en los llamados consejos de cuenca. Metodológicamente, se recurre a la etnografía para describir el funcionamiento de estos consejos e identificar las fallas en su implementación. Se concluye que, para fortalecer la gobernanza del agua, hace falta la acción colectiva sobre las reglas informales, adicionalmente a la incidencia sobre las formales.

Palabras clave: gestión del agua, consejo de cuenca, reglas informales, políticas públicas, río Santiago.

\begin{abstract}
This article analyzes the implementation of a water policy on the Santiago River (Mexico), especially about social participation in water management, highlighting the role of informal rules in so called basin councils. Methodologically, ethnography is used to describe the functioning of this councils and identify the flaws in its implementation. It's concluded that, in order to strengthen the governance of water, collective action on informal rules is required, in addition to the incidence on formal rules.
\end{abstract}

Keywords: Water management, basin councils, informal rules, public policies, Santiago River.

\footnotetext{
-Profesor-Investigador en la Universidad del Valle de México, campus Guadalajara Sur, México. ORCID: http://orcid.org/0000-000 I-6946-4596_enlujan@hotmail.com

- Profesora-Investigadora en el Centro de Investigación en Ciencias de la Información Geoespacial (Centro Geo), campus Aguascalientes,y Cátedra Conacyt, México. ORCID: http:// orcid.org/0000-0002-9070-7223_mfuerte@centrogeo.edu.mx

Fecha de recepción:09 de abril de 2018. Fecha de aceptación: 13 de agosto de 2018.
} 


\section{Introducción}

El tema de los consejos de cuenca ha sido estudiado desde diversas perspectivas (Guzmán Arroyo, 2001; Flores Elizondo, 2012; Bravo, 2005; Palerm y Rodríguez, 2005; Vargas y Mollard, 2005; Sánchez, 2010; Sancho y Parrado, 2004; Galindo Sosa, 2009). Sin embargo, existen pocas investigaciones que ilustren las dinámicas cotidianas de estas organizaciones. En esta investigación, se aborda el funcionamiento de un consejo de cuenca concreto, y se destaca la centralidad de los actores que participan estratégicamente en las organizaciones sociales (Crozier y Friedberg, 1990). Particularmente, el estudio está orientado a conocer un modelo de política pública desde la perspectiva de los actores involucrados (Long, 2007).

El objetivo es ilustrar el proceso de implementación de un programa de Gobierno hecho a partir de un juego estratégico en el que los actores sociales involucran sus propios intereses y valores para incidir en el gobierno del agua. La pregunta de investigación ha sido ¿cómo las decisiones al interior de los consejos de cuenca impactan en el modelo de una política pública? Se busca mostrar que, más allá del diseño formal, las normas informales importan en estos procesos, y en el caso del consejo de cuenca permiten un manejo discrecional del agua. Para ello, se utiliza como caso el Consejo de Cuenca del río Santiago (México).

En el primer apartado del texto, se presenta la perspectiva teórica y metodológica desde la cual se hace el análisis de este consejo de cuenca. En el segundo apartado, se incluye una descripción de la cuenca en cuestión y los antecedentes de su consejo, recopilando la literatura central sobre este tema. En el tercer apartado, se presentan los resultados, que ilustran el funcionamiento cotidiano del consejo, y se concluye con una discusión en la cual se insiste en la impor-

\section{2}


tancia de las reglas informales y en la necesidad de incidir en ellas a partir de la acción colectiva.

Resulta fundamental comprender las formas en que interactúan los diferentes actores y tomadores de decisiones. Los modelos de política son construcciones conceptuales que surgen de una disputa discursiva para nombrar y construir problemas susceptibles de ser incorporados en una agenda pública, y en ese sentido reflejan los intereses estratégicos de los actores involucrados, por lo que importa conocer a dichos actores en nuestro caso de estudio en tanto estos construyen los diferentes escenarios del agua y las perspectivas desde las cuales deciden abordar los problemas públicos.

\section{Problemas, actores y modelos de política del agua}

Contra la creencia popular de que los problemas públicos son evidentes por sí mismos, desde el análisis de políticas públicas se afirma que los problemas no existen per se, y que su existencia es más supuesta que real (Aguilar, 1993, p. 57). Es decir, no todas las situaciones lamentables que existen en nuestra sociedad son percibidas como problemas, e incluso no todos los problemas que son percibidos como tales alcanzan su inclusión en la categoría de temas a debatir para incorporarlos en una agenda pública (Olavarría, 2007).

Los problemas públicos, entonces, son elaboraciones humanas (Becker, 1995). Se trata de construcciones conceptuales que surgen de condiciones sociales incorporadas en la agenda de actores con la capacidad de incidir en la discusión pública. En este proceso de construcción de agendas y toma de decisiones, los Gobiernos establecen normas, acciones y estrategias para resolver problemas públicos, las cuales reciben el nombre de políticas públicas (Olavarría, 2007, p. 93). En la formación y aplicación de una política pública, la 
parte central será no sólo decidir cuáles problemas serán tomados en consideración, sino, principalmente, cómo serán abordados y descritos y atendidos (Aguilar, 1993; Merino, Arellano Gault, Cejudo, et al., 2010).

Las definiciones de los problemas "no sólo estructuran y encuadran las elecciones posteriores de la política; también sirven para afirmar una concepción particular de la realidad" (Cobb y Elder, 1974, p. 115). La perspectiva desde la que se define un problema también definirá los instrumentos de acción y a la propia política (Olavarría, 2007). De esa manera, el análisis conceptual de las políticas públicas en realidad es un análisis de las motivaciones de los actores que las definen (Aguilar, 1993; Cejudo, 2008).

En México, para atender el problema del agua, oficialmente se ha adoptado el llamado modelo GIRH o de la gestión integral de los recursos hídricos (Grupo de Trabajo Buena Gobernanza, 2012). Este modelo puede definirse como

un proceso que promueve la gestión y el desarrollo coordinado del agua, la tierra y los recursos relacionados, con el fin de maximizar el bienestar social y económico de manera equitativa, sin comprometer la sostenibilidad de los ecosistemas vitales (Global Water Partnership, 2013, p. 16).

El modelo se trata más bien de una caja de herramientas que pretende orientar la implementación de políticas públicas a partir de cuatro principios: 1) el agua es un recurso finito y vulnerable, esencial para sostener la vida, el desarrollo y el medio ambiente; 2) el aprovechamiento y la gestión del agua deben inspirarse en un enfoque basado en la participación de los usuarios, los planificadores y los responsables de las políticas en todos los niveles; 3) la mujer desempeña un papel fundamental en el abastecimiento, la gestión y la protección del agua; 4) el agua es un bien público y posee 
un valor económico y social en todos sus diversos usos, que compiten entre sí (Global Water Partnership, 2009).

Este modelo se adoptó en México en un contexto de grandes transformaciones sociales que incluían una mayor incorporación de la sociedad en los asuntos del Estado, la demanda social de más y mejor gobernanza y la transformación del sistema político tradicional. Además, en el contexto internacional, la adopción permitió que los Gobiernos mexicanos adquirieran legitimidad en la comunidad global interesada en la gestión y manejo de los recursos naturales (Ruiz, 2015).

Ahora bien, el modelo GIRH promueve el uso sustentable de las cuencas a partir de una gestión participativa, eficaz y equitativa (Global Water Partnership, 2009), lo cual pareciera contradictorio con la realidad de una gestión mercantilista y vertical, propia del Gobierno mexicano. Esa contradicción entre el diseño y la implementación de una política es lo que interesa estudiar.

Para ello, se ha elegido a la etnografía para la recolección y el análisis de la información. La etnografía es una técnica cualitativa con la que se busca construir una interpretación de la realidad a partir de la participación, la observación y las entrevistas que hace el investigador en un grupo bajo estudio (Guber, 2001). Esto se adecuó con el objetivo de la investigación planteada, que fue identificar los problemas públicos que los integrantes de un consejo de cuenca en México perciben como tales, y las formas concretas en que son definidos y atendidos esos problemas.

Para empezar, se definieron dos etapas para la recolección y análisis de la información: incursión y comprensión. Luego, en la etapa de incursión, se aplicó la observación no participante y la investigación documental (Guber, 2001). Se asistió a las reuniones del Consejo de Cuenca del río Santiago y sus órganos auxiliares y funcionales realizadas entre julio de 2011 y mayo de 2013, se identificaron perfiles 
de los actores participantes y fue generado un listado de los temas en su agenda (Maldonado y Casar, 2008). Siguiendo las recomendaciones de Santander (2011), se recurrió también a una revisión de los documentos resguardados en el archivo histórico del consejo, particularmente las actas de las reuniones, registros de asistencia y otros materiales conservados, como oficios, folletos, presentaciones, etc.

Finalmente, en la etapa de comprensión, se realizó observación participante de las sesiones del consejo entre julio de 2013 y diciembre de 2015, al tiempo que se elaboró una revisión hemerográfica para identificar los temas presentes en la agenda pública durante dicho periodo. También, fue aplicado un cuestionario para medir la percepción de los actores respecto al desempeño de la Comisión Nacional del Agua (Conagua) en el consejo de cuenca (Soares y Murillo, 2013) y se realizaron entrevistas a profundidad.

\section{La cuenca del río Santiago y el programa de consejos de cuenca}

El río Santiago recorre el centro-occidente de México y su cuenca está incluida en el territorio total o parcial de siete estados. Estrictamente hablando, el río Santiago es continuación del Lerma, aunque administrativamente se consideran cuencas separadas (Comisión Nacional del Agua, 2010). Luego de su nacimiento en el Lago de Chapala, el río Santiago recibe en su margen derecho el caudal de los cauces tributarios formados principalmente en las serranías de los estados de San Luis Potosí, Guanajuato, Aguascalientes, Zacatecas y Durango (ver: Figura 1). Su recorrido termina al desembocar en el océano Pacífico, en el estado de Nayarit, y sus tributarios más importantes son el río Verde, el río Juchipila, el río Bolaños y el río Huaynamota, los cuales forman subcuencas totalmente independientes (Comisión Nacional del Agua, 2015).

\section{6}


Existe una confusión en la literatura no especializada que tiende a reducir la cuenca del río Santiago sólo a su tramo entre el Lago de Chapala y la ciudad de Guadalajara, que es uno de los puntos del trayecto más poblados, industrializados y contaminados. En realidad, la cuenca tiene una mayor dimensión en términos físicos y administrativos, lo que contribuye a hacer más compleja su problemática, que incluye además otros aspectos relacionados con la desertificación, la deforestación, el cambio climático, la sequía, los patrones de poblamiento, la escasa infraestructura y el impacto de la estructura demográfica.

En la cuenca del Santiago se contabilizan cuarenta y siete acuíferos, de los cuales ocho se encuentran en condiciones de sobreexplotación. Entre estos últimos, destacan los que se localizan en el estado de Aguascalientes, en la zona metropolitana de Guadalajara y en el valle agrícola de Jerez, en Zacatecas (Comisión Nacional del Agua, 2015). El grado de presión sobre el recurso hídrico es fuerte, y la disponibilidad del agua superficial está restringida por las declaratorias de veda emitidas mediante decretos presidenciales en los años de 1931, 1947, 1954 y 1969 (Comisión Nacional del Agua, 2015), las cuales fueron modificadas recientemente por otro decreto presidencial cuyas implicaciones no son de interés para el presente artículo.

En su conjunto, la cuenca del río Santiago tiene una superficie de $76720 \mathrm{~km}^{2}$, y la habitan alrededor de ocho millones de personas, distribuidas en 11081 localidades dentro de ciento veinticuatro municipios (Comisión Nacional del Agua, 2015). El 87\% de esta población se concentra en localidades urbanas, siendo las más importantes tres zonas metropolitanas: Guadalajara, Aguascalientes y Tepic (Instituto Nacional de Estadística y Geografía, 2015). Para atender los problemas en la cuenca y buscar soluciones coordinadas, fue promovida la instalación del consejo de cuenca, lo que se logró en julio de 1999. 
Figura 1. Subcuencas de la región hidrológica denominada Río Santiago

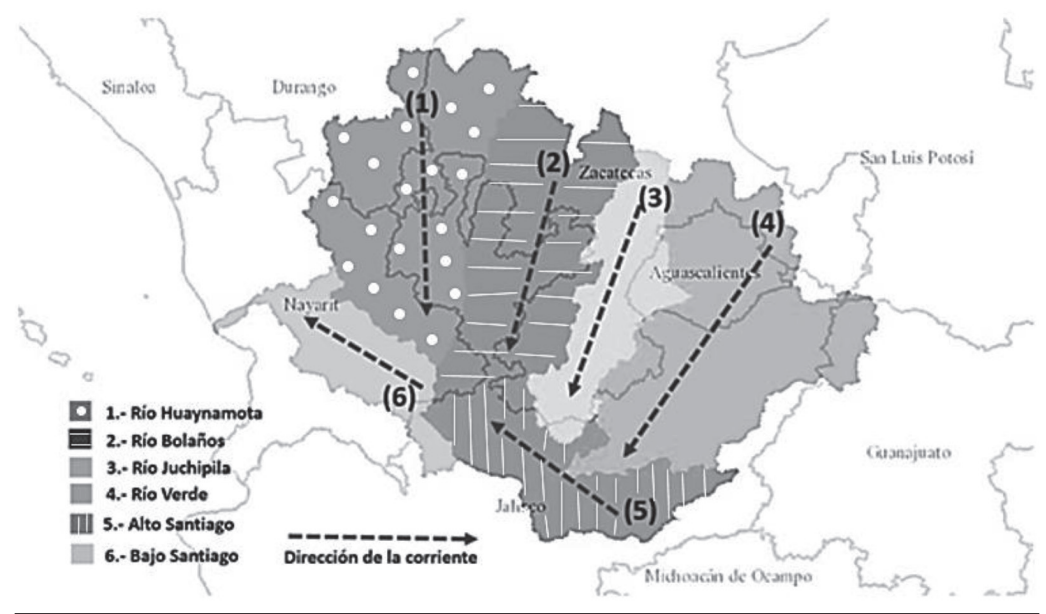

Fuente: elaboración propia con base en Instituto Nacional de Estadística y Geografía (1988).

$\mathrm{Al}$ igual que los otros consejos de cuencas que existen en el país, el del río Santiago ha tenido fallas y obstáculos tanto en su diseño como en su implementación (Parra-Armenta y Salazar, 2018). Idealmente, esta organización sería el espacio en donde se coordinarían la autoridad en los tres órdenes de Gobierno y la sociedad organizada (Grupo de Trabajo Buena Gobernanza, 2012), y en donde, de manera participativa, se gestionarían los recursos hídricos integralmente. Sin embargo, desde su diseño formal, privaron las visiones autoritarias sobre estos consejos y no se les reconocieron atribuciones formales respecto a ejecutar, realizar, ejercer u ordenar, dejándolos sólo como un espacio consultivo (Martínez, Martínez, y López, 2014).

La Conagua es la responsable de la instalación, organización y funcionamiento de los consejos de cuencas. Para ello, cuenta con recursos y una importante estructura administrativa que piramidalmente inicia en las oficinas 
centrales, incluye a los organismos de cuenca, y termina en las direcciones locales, dependientes todos de la misma dirección general. Adicionalmente a los recursos humanos, materiales y financieros que posee la Conagua para sostener esta estructura orgánica, existe el programa U015 Consejos de Cuenca, que es federal, administrado por la propia Conagua pero ejecutado por los Gobiernos locales mediante convenios de colaboración (Auditoría Superior de la Federación, 2015).

Los recursos presupuestales del programa U015 son repartidos discrecionalmente por la Conagua a los veintiséis consejos de cuenca establecidos en todo el territorio. Este monto, al que se debe sumar una aportación de los Gobiernos locales, ha sido asignado y ejercido año tras año con el objetivo de apoyar la instalación de los consejos de cuenca y fortalecer su integración y operación. Tan sólo en el 2015, se contó con una bolsa de noventa y cinco millones de pesos, en donde 47.5 millones correspondieron al aporte del Gobierno federal y una cantidad similar a las aportaciones de los Gobiernos estatales y municipales que participaron (Secretaría de Hacienda y Crédito Público, 2016).

Para la tarea de instalar y organizar a los consejos de cuenca, la Conagua propuso una estrategia de acción para un horizonte de cinco años, misma que consideraba cuatro etapas diferentes: gestación, instalación, consolidación y operación plena (Comisión Nacional del Agua, 2010). El primer consejo de cuenca se instaló en enero de 1993 (el Consejo de Cuenca Lerma-Chapala), y transcurridos veinticinco años aún no puede ser reconocida una participación efectiva de los consejos de cuenca en la gestión y gobierno del agua. Los recursos, los funcionarios y la estructura destinada a promover la instalación, el fortalecimiento y la operación de los consejos de cuenca han sido insuficientes e inadecuados. Incluso, desde el Gobierno federal se reconoce que no se ha alcanzado una adecuada integración ni fortalecimiento de 
los consejos para asegurar la representatividad y atención de las necesidades de todos los usuarios del agua, así como su plena participación en la toma de decisiones (Auditoría Superior de la Federación, 2015).

La Auditoría Superior de la Federación (2015) ha hecho a la Conagua catorce observaciones y veintidós recomendaciones al desempeño. Entre ellas, ha pedido incluir en la política nacional hídrica la pluralidad de intereses, demandas y necesidades respecto de las cuencas hidrológicas. Específicamente, la auditoría ha hecho recomendaciones dirigidas a consolidar la participación de los consejos de cuenca, establecer mecanismos de coordinación para elaborar sistemas regionales de información, realizar estudios con la participación de los consejos y promover la integración en ellos de los integrantes de la sociedad organizada (Auditoría Superior de la Federación, 2015).

Pasadas más de dos décadas de su implementación, y gastados alrededor de dos mil millones de pesos, algunos consejos de cuenca están apenas en su proceso de instalación, ninguno cuenta con plena autonomía operativa y financiera, y en menor o mayor medida todos están subordinados a la Conagua. Se encuentran fallas en ellos, como su no integración conforme a lo previsto en la ley (26\% de los consejos de cuenca), no contar con reglas de organización (30\%), no haber electo a un presidente (50\%), no tener un programa de gestión (44\%), y el más grave y paradójico: no reunirse para sesionar (46\%) (Auditoría Superior de la Federación, 2015).

En la literatura especializada, además de la falla en el diseño institucional de los consejos de cuenca, en el sentido de que formalmente son sólo un espacio consultivo sin ninguna facultad vinculante, han sido identificados también una escasa representatividad de los actores que participan en los consejos, la disparidad en el acceso a recursos e información entre actores que se pretenden iguales, un proceso

\section{0}


pobre de toma de decisiones (limitado a la validación de lo que dicta la autoridad central), y un carácter excluyente de los funcionarios públicos en los organismos de la cuenca (Barkin y Klooster, 2006; Barreda, 2006; Bravo, 2005; Flores Elizondo, 2012; Galindo Sosa, 2009; Guzmán Arroyo, 2001; Martínez, Martínez, y López, 2014; Mussetta, 2009; Palerm y Rodríguez, 2005; Sánchez, 2010; Sancho y Parrado, 2004; Vargas y Mollard, 2005).

Estas críticas al funcionamiento del programa describen una realidad en donde hay no sólo problemas de diseño, sino también una falla en la adopción de un modelo de gestión del agua identificable con el modelo GIRH, cuyos principios, como ya se mencionó, postulan un enfoque participativo, democrático, eficiente y equitativo (Soares y Vargas, 2008).

\section{El funcionamiento del consejo de cuenca}

La revisión documental de las sesiones del Consejo de Cuenca del río Santiago y de las de sus órganos auxiliares permite inferir al menos tres periodos en el funcionamiento del consejo: una fase de gestación, una fase de consolidación y una fase de destrucción y desmantelamiento.

El primer periodo dura tres años, y va de la primera a la segunda reunión del consejo (años 1999-2002). Es un periodo en el que hubo reuniones de los órganos auxiliares sólo para definir el tipo de organismo que sería el consejo, sus alcances legales, la autonomía y autoridad que se le reconocería, etc. (Actas Cocurs, 2016). Es posible interpretarlo como un periodo de socialización de normas y definición de agendas, que duró cerca de cuatro años.

El periodo de consolidación va de la segunda a la décima sesión (años 2002-2012), y se caracteriza porque en él se procuró cumplir con la norma formal en cuanto a la periodicidad de las reuniones, se comenzaron a abordar gradualmente temas específicos de la gestión del agua, se 
integraron órganos auxiliares para atender problemas locales, y se generaron grupos de trabajo para otros problemas concretos. Poco a poco, el consejo se consolidó e incrementó su número de integrantes, esto para atender la reforma a la ley del año 2004. Incluso, se eligió a un presidente del consejo ajeno a la Conagua (Actas Cocurs, 2016). Este periodo duró cerca de diez años.

El tercer periodo va del año 2012 hasta la actualidad. Según está prevista en la estrategia de implementación, esta sería la etapa de funcionamiento pleno, pero en el caso del Consejo de Cuenca del río Santiago ha significado una etapa de declive, sin reuniones del consejo de cuenca realizadas. Es una etapa de desmantelamiento y destrucción, cuya explicación debe buscarse en un cambio en la coalición gobernante en el país y su falta de interés en el tema de la participación, la transparencia y la rendición de cuentas (entrevista personal, 2015a). Incluso, en esta etapa las voces que se perciben como críticas al interior del consejo son marginadas o apartadas de la organización (entrevista personal, 2015a).

\section{I. La agenda en el consejo de cuenca}

Los actores que integran el Consejo de Cuenca del río Santiago fueron capaces de identificar diversos problemas del agua, pero, incluso en su etapa más productiva, el consejo no transitó hacia un programa de gestión participativo y eficiente que tuviera un impacto real en la gestión del recurso: en los acuerdos suscritos y en su seguimiento, prevalece el deseo de los integrantes de limitarse al cumplimiento de disposiciones normativas (el perfeccionamiento del consejo, la validación de estudios, la elección de usuarios, la elección del presidente, la aprobación de las reglas internas del consejo, etc.), y son pocos los acuerdos orientados a la atención de la problemática hídrica (Actas Cocurs, 2016). 
La revisión de las actas suscritas en cada una de las diez sesiones realizadas durante el periodo de análisis muestra que de las veinticinco responsabilidades descritas en la ley (artículo 13, bis 4) como facultades de los consejos de cuenca (Congreso de los Estados Unidos Mexicanos, 2016), existe evidencia del cumplimiento de sólo diez (Actas Cocurs, 2016). Entre las responsabilidades omitidas destacan: concertar las prioridades de uso del agua; participar en la definición de los objetivos generales y los criterios para la formulación de los programas de gestión del agua; coordinar y complementar inversiones en materia hídrica; y conocer oportuna y fidedignamente la información y documentación referente a la disponibilidad, cantidad, calidad, usos y derechos registrados del agua, así como los tópicos y parámetros de mayor relevancia en materia de recursos hídricos y su gestión.

En contraste, en la agenda del consejo se repite rutinariamente el cumplimiento de normas formales, administrativas, rutinarias y protocolarias (Actas Cocurs, 2016). A veces, la estrategia percibida es derivar los temas álgidos hacia comisiones de cuenca o grupos de trabajo, en donde formalmente serán atendidos pero no se acordarán acciones conjuntas con la participación de sus integrantes (entrevista personal, 2015b). Así ocurre, por ejemplo, con el tema del saneamiento, con el proyecto educativo Descubre una cuenca; río Santiago, con los grupos de trabajo de sustentabilidad y uso eficiente del agua, y con algunas comisiones de cuenca instaladas en los espacios locales (entrevista personal, 2015c).

En los grupos de trabajo del consejo de cuenca no hay participación de los usuarios, sólo de representantes estatales y de Conagua, quienes se limitan a sumar cifras por estado, a describir sus actividades cotidianas y a generar presentaciones en donde agregan los números de cada dependencia. 


\subsection{Los actores del consejo de cuenca}

Se identificaron tres tipos de actores en el Consejo de Cuenca del río Santiago: activos, pasivos y ausentes. Los actores activos son la Conagua y los Gobiernos de los estados; los actores ausentes son las dependencias federales y los Gobiernos municipales, que no participan del consejo pero podrían hacerlo si su presencia o su voto fuera requerido; y los actores pasivos son los usuarios, la academia y la sociedad organizada, limitados a validar los acuerdos que se tomaron previamente.

En las reuniones de Consejo a las que se asistió a observar (sesiones nueve y diez), el orden del día se redujo a una serie de mensajes, informes y saludos en donde los actores importantes (es decir, los activos) anunciaron las nuevas políticas y acciones respecto a la gestión del agua en la cuenca. La participación de los actores pasivos fue nula, limitada a validar los acuerdos levantando la mano cuando se les solicitó (Actas Cocurs, 2016).

La Conagua trata de usar al consejo de cuenca para sus intereses estratégicos, y el resto de actores que integran esta organización hace lo propio, de manera que cuando se les convoca a una reunión asisten a ella porque así pueden estar cerca de gobernadores, delegados de las diferentes secretarías federales, presidentes municipales y otros funcionarios, una cercanía que es valiosa para el logro de sus intereses (entrevista personal, 2013a).

Formalmente, las reuniones las organiza el Gobierno del estado anfitrión (se trata de reuniones que organiza un Gobierno estatal diferente cada vez, de entre los involucrados en la cuenca del río), pero los equipos de logística estatal y federal se reúnen para empatar agendas y resolver asuntos de protocolo (como los turnos en el uso de la palabra o la disposición de las sillas en el presídium). Todo está acordado de manera previa: se tiene un orden del día 
riguroso y no hay lugar para espontáneos ni improvisadores (entrevista personal, 2013b).

El Gobierno federal informa, y lo mismo hacen los Gobiernos estatales, sobre montos de inversiones, población beneficiada, catálogo de acciones y proyectos, etc., pero ninguno de los participantes somete a la consideración del consejo el presupuesto programado, las prioridades de inversión, la distribución de recursos, las acciones específicas o los costos de las obras. Tampoco se presenta información acerca del estado del agua en la cuenca, ni se toman acuerdos sobre su distribución o las prioridades de uso (según información recabada en diarios de campo).

\subsection{Percepciones sobre el programa de consejos de cuenca}

El enfoque top-down desde el que se implementó el programa de consejos de cuenca permea a la organización y define sus prácticas informales. La visión que prevalece supone que las capas superiores son capaces de generar decisiones casi perfectas, de organizar la mejor forma de llevar a cabo estas decisiones, y de ejecutarlas con precisión hasta producir los resultados esperados (Revuelta, 2007).

Como una visión racional ideal, el enfoque top-down hace alarde de eficacia y eficiencia y afirma que el Estado es un ente organizado y racional (Maldonado, Valera, y Palma, 2013). Se asume el supuesto de que desde arriba de la pirámide existe la capacidad de establecer objetivos claros, estructurar las órdenes hacia abajo en una agenda debidamente prevista y asegurar los recursos y apoyos necesarios, lo mismo que se confía en el establecimiento de un sistema de comunicación que garantice el descenso de las órdenes hacia los responsables y la retroalimentación hacia el poder central.

Sin embargo, tal ideal no existe. En realidad, las burocracias actúan de manera contingente, rutinaria, pragmática, sin claridad en los objetivos; son erráticas en las acciones 
y dispersas en sus intereses (Maldonado, Valera, y Palma, 2013, p. 24). Es por eso que en la agenda del consejo de cuenca, para las organizaciones burocráticas "predomina la contingencia y las acciones erráticas, las respuestas rutinarias y las decisiones pragmáticas" (Maldonado, Valera, y Palma, 2013, p. 27).

En el consejo de cuenca no existe un programa de gestión, no hay metas ni objetivos establecidos, no existe un calendario de supervisión y seguimiento y no hay participación ni coordinación en el uso de la cuenca y de sus recursos. Lo que se observa es una agenda supeditada a los intereses de la Conagua, a lo que pide el superior, a la percepción que tienen los funcionarios sobre lo mejor y a su discrecionalidad para interpretar las prioridades, es decir, a sus intereses estratégicos (Actas Cocurs, 2016). Son estos funcionarios públicos quienes convocan a la reunión y la cancelan si les parece oportuno, los que fijan el orden del día o lo cambian según lo desean; son ellos quienes pueden definir la sede, la logística, los invitados, los temas y hasta el código de vestimenta. Y si lo deciden, también pueden congelar a la organización y llevarla a la inactividad, excluyendo de la discusión cualquier tema que no les guste (entrevista personal, 2015d).

Es así como esta organización funciona: con una agenda vertical. Pueden pasar años sin que se reúna, aun cuando existan temas que desborden la agenda pública, pero también en cualquier momento la Conagua puede hacer una reunión emergente y presionar una discusión, o lograr que se validen acciones que afectan a toda la cuenca simplemente con una consulta a mano alzada: establecer medidas en contra de la sequía, avalar el programa hídrico regional o conocer convenios en donde se decretan transvases de agua hacia otras cuencas (entrevista personal, 2015d).

A pesar de este panorama negativo, se destaca que el 80\% de los integrantes usuarios tiene una percepción positiva 
del consejo de cuenca. Mediante un cuestionario diseñado para medir las percepciones de los usuarios respecto al desempeño de la Conagua, se evaluaron tres cosas: la satisfacción respecto a los temas que formalmente le corresponde atender a la Conagua (ver: Tabla 1); la percepción (negativa o positiva) respecto a la apertura y disposición de la Conagua para atender la participación social en la gestión del agua (ver: Tabla 2); y la percepción respecto a lo que la Conagua hace para fomentar la participación democrática (ver: Tabla 3).

\section{Tabla 1. Satisfacción de los vocales usuarios con el desempeño de Conagua}

Tópico

Estoy satisfecho con el tiempo y la forma en que se me entregó la acreditación como miembro del consejo de cuenca.

Estoy satisfecho con el seguimiento de acuerdos que se hace de cada sesión, y con el informe del avance de los mismos. Estoy satisfecho con los espacios y la forma en que se realizan las diversas sesiones de este consejo de cuenca. Estoy satisfecho con el tiempo y forma que se emiten las convocatorias para asistir a las diversas sesiones del consejo de la cuenca.

Estoy satisfecho con el trato que he recibido por parte de personal de la Conagua.

Estoy satisfecho con la forma en que se define el orden del día y los puntos a tratar en las diversas sesiones.

Estoy satisfecho con la forma en que se me informa de las tareas y actividades que se realizan en el consejo de la cuenca.
Puntaje (escala de 1 a 10)

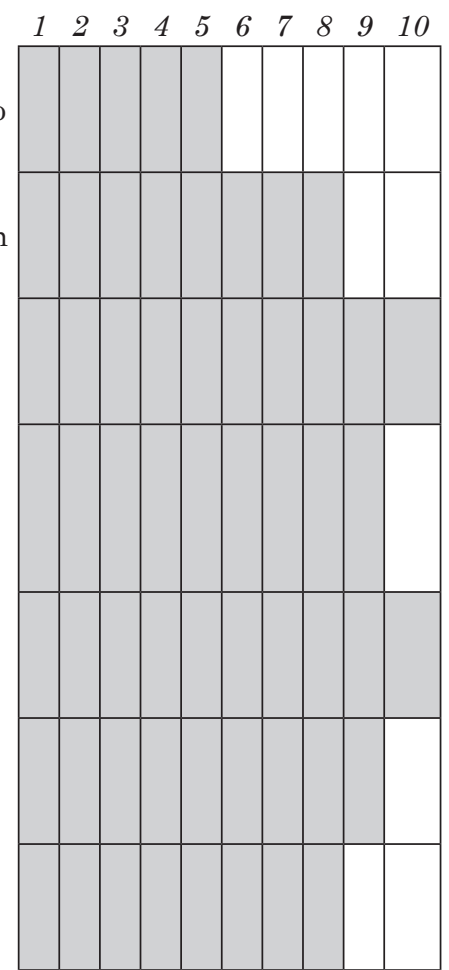


Tópico

Estoy satisfecho con la forma en que se han desahogado y resuelto las propuestas y consideraciones que yo he presentado. Estoy satisfecho con el desempeño de la Secretaría Técnica en las sesiones y actividades del consejo.

Estoy satisfecho con los resultados que se han alcanzado en el consejo de cuenca.

Fuente: elaboración propia a partir de cuestionarios.

\section{Tabla 2. Percepción de los usuarios sobre la Conagua respecto a la participación social}

Tópico

La Secretaría Técnica es muy cerrada en cuanto a los temas que se traten en el consejo de cuenca.

La Secretaría Técnica se percibe muy comprometida con el consejo de la cuenca.

La Secretaría Técnica promueve acciones que son sustentables y respetuosas del medio ambiente.

Cuando la Secretaría Técnica asume una tarea, es muy ineficiente en su cumplimiento.

La Secretaría Técnica promueve la participación en la programación hídrica en la cuenca.

El trabajo que se hace en este consejo de cuenca ha sido intrascendente.

El trabajo de la Secretaría Técnica es muy transparente, y se nos informa de manera constante y sistemática.

La elección de vocales es democrática y representativa.
Puntaje (escala de 1 a 10)

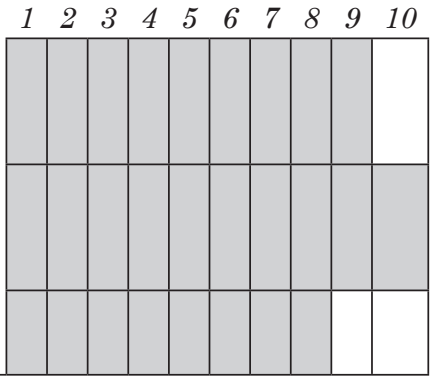

Puntaje (escala de 1 a 10)

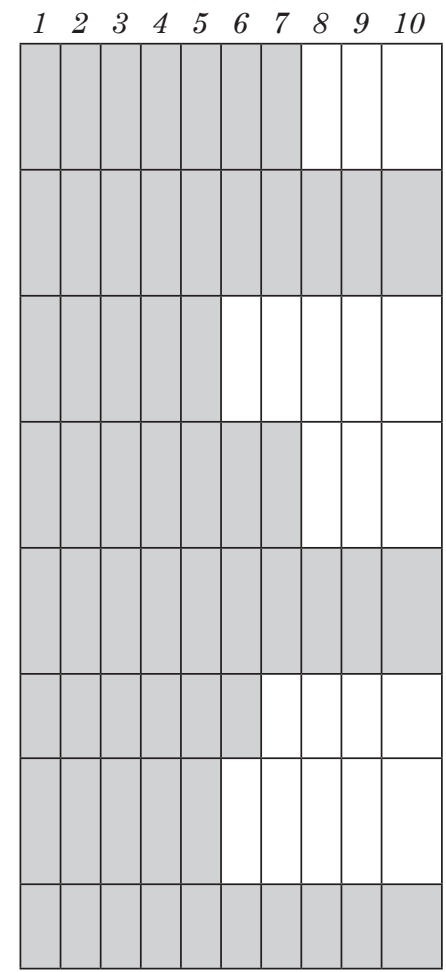

\section{8}


Tópico

Nunca he sido consultado respecto a la programación hídrica en la cuenca. Pienso que los problemas más importantes del agua en la cuenca sí se discuten en el consejo de la cuenca.
Puntaje (escala de 1 a 10)

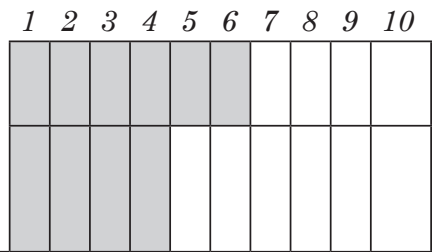

Fuente: elaboración propia a partir de cuestionarios.

En tres rubros, la Conagua resulta reprobada: 1) la percepción respecto a la importancia de los temas tratados (la calificación más baja obtenida), 2) la transparencia en el consejo, y 3) la sustentabilidad de las soluciones que promueve. Además, la Conagua fue evaluada con la calificación mínima aprobatoria en el tópico que se refiere a la trascendencia del trabajo que se hace en el consejo de cuenca, lo que indica que incluso los usuarios más satisfechos perciben que la organización no ha logrado impactar de manera trascendente en la gestión del agua (ver: Tabla 3). En general, la percepción sobre su trabajo es que ha sido bueno.

Tabla 3. Percepción de los usuarios respecto a la democracia en el consejo de cuenca

Tópico

En mi opinión, los puntos que la Secretaría Técnica incluye en el orden del día de cada reunión siempre se someten a la consideración del consejo de cuenca, y cualquiera puede proponer algún punto de interés.

La Secretaría Técnica es muy eficiente en el seguimiento de acuerdos y en la gestión de resultados en aquellas tareas que le son encomendadas.
Puntaje (escala de 1 a 10)

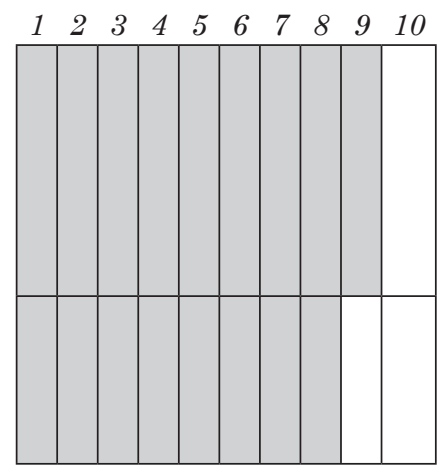


Tópico

Puntaje (escala de 1 a 10)

En el consejo de cuenca, la Secretaría Técnica promueve ampliamente la participación de los representantes usuarios de agua y de la sociedad civil. La Secretaría Técnica ha fomentado la existencia de un inventario completo de estudios y proyectos relacionados con la cuenca, y este inventario está accesible para cualquiera que lo pueda consultar. Las convocatorias para la elección de los vocales en el consejo de cuenca siempre se han hecho de la manera más amplia y democrática posible.

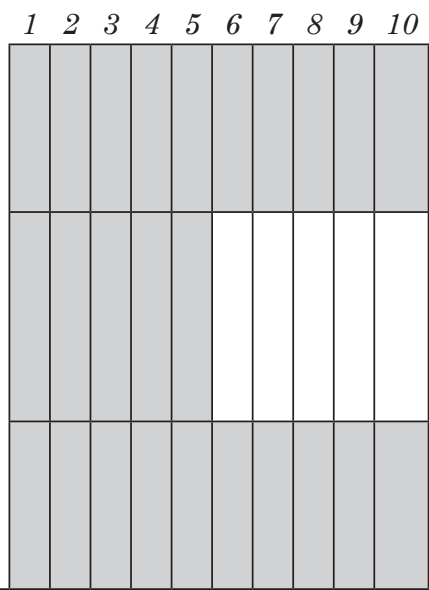

Fuente: elaboración propia a partir de cuestionarios.

El cuestionario aplicado sólo captó la percepción respecto a la parte formal, que es responsabilidad de la Conagua. En ese aspecto, la evaluación que se hace es positiva, pero es importante señalar que en las entrevistas sostenidas con los usuarios desde diferentes posturas y con sus propias palabras se mencionan situaciones y rutinas que les disgustan o perciben como excluyentes, poco eficientes o antidemocráticas. El cuestionario se aplicó a los vocales usuarios de todos los usos, pero el de uso público urbano decidió no responder.

Es decir, los actores involucrados en el consejo de cuenca analizado tienen una percepción similar a la que se tiene en la Auditoría Superior de la Federación, y muy parecida también a la opinión de algunos funcionarios en los Gobiernos estatales y a lo que constantemente se está denunciando desde la academia: si bien es cierto que el programa de consejos de cuenca formalmente funciona en todo el país como una herramienta para la gestión del agua, es claro que existen fallas tanto en su diseño como en su implementación que impiden obtener los resultados esperados de él. 


\section{Funcionamiento ideal y funcionamiento real}

Como el resto de organizaciones de cuenca que existen en el país, el Consejo de Cuenca del río Santiago está formalmente muy limitado en sus alcances. Funcionarios de Conagua que formularon este señalamiento relataron que esas limitaciones no surgieron en sus oficinas, sino que fueron agregándose en la búsqueda de consensos con el Poder Legislativo y por presión de Gobiernos estatales que percibieron como un atentado a su integridad y soberanía la división del país en cuencas hidrográficas (entrevista personal, 2015b).

Cualquiera que sea la realidad, además de las limitaciones en su diseño formal, se encontró que la implementación del consejo estuvo condicionada por dos creencias de los actores gubernamentales: una manifestada en un discurso tecnocrático, desde el cual la participación no se asume como un bien positivo, sino como un obstáculo para las decisiones técnicas que, por definición, siempre son mejores; y una manifestada en un discurso político, en el cual se reproducen prácticas de representación política corporativa y excluyente. Desde estas creencias, se buscó incorporar al consejo de cuenca actores que formalmente cubrieran el perfil requerido, pero que tuvieran afinidad ideológica o política o, incluso, relaciones de amistad o lazos familiares, en lugar de privilegiar una promoción abierta y democrática para la integración de estos espacios. Estas prácticas aseguraban organizaciones afines, dóciles y maleables (entrevista personal, 2015b). 
Tabla 4. Diferencias entre el marco formal y las prácticas informales en el Consejo de Cuenca del río Santiago

\section{Marco formal}

El consejo de cuenca es un espacio de coordinación

Se integra por usuarios

representantes de todos los usos del agua en la cuenca

Tiene diversas funciones enlistadas en la Ley de Aguas Nacionales

Se promueve la gestión integral de recursos

Se deben reunir al menos de manera anual

Atiende la problemática de la cuenca

La participación busca la sustentabilidad, la equidad y la eficiencia

Se proponen soluciones técnicas para que el consejo adopte la mejor
Práctica informal

Es una organización vertical

Los usuarios no son sometidos a mecanismos de representación

Se limita a validar lo que Conagua solicita

Sólo dos de treinta y siete integrantes son mujeres

Tienen tres años sin sesionar en pleno

Se evita tratar los problemas de la cuenca

Se actúa de manera discrecional, y tanto la sustentabilidad como la equidad no son importantes Se opera políticamente para que no se consideren alternativas a lo que Conagua ha decidido

Fuente: elaboración propia a partir de la observación participante.

Como puede observarse en la Tabla 4, la participación fue asumida en este consejo de cuenca como un requisito a cumplirse sólo en el plano formal, y no como un mecanismo para el mejor gobierno del agua en la cuenca. En ese sentido, la propuesta innovadora del modelo GIRH fue pervertida y limitada por las prácticas políticas cercanas al corporativismo, el compadrazgo, el caciquismo y el uso patrimonial de los bienes públicos. El resultado fue el fortalecimiento de un modelo excluyente, mercantilista y discrecional, en lugar de una política del agua participativa, sustentable y equitativa.

La gran promesa de la transformación en la gestión del agua que significó la primera Ley de Aguas Nacionales, lo 
mismo que sus posteriores reformas, se redujo a: 1) decretar la corresponsabilidad de los tres niveles de Gobierno más como mecanismo para diluir responsabilidades que como herramienta de coordinación; 2) fragmentar la acción colectiva para limitarla a los aspectos administrativos de la cuenca; y 3) supeditar las decisiones técnicas a los intereses políticos, coludidos con los económicos. Hoy, la gestión integral del agua es sólo parte de un discurso que se materializa en el abandono de los ecosistemas, el deterioro de la cuenca y la reducción de la calidad de vida para las personas en los espacios locales donde no existe una acción colectiva organizada.

\section{Conclusiones}

El tradicional legalismo en México (Narváez, 2005) ha llevado a diversos académicos y actores sociales a centrar su crítica respecto al diseño formal de la política pública hídrica en sus fallas observables, y a demandar un nuevo documento normativo al respecto. Sin embargo, el propósito de este artículo ha sido demostrar que las reglas informales también importan, y que, independientemente de la norma formal, existe un juego estratégico de intereses que da como resultado una forma particular del gobierno del agua en las cuencas.

En el caso de la cuenca del río Santiago, los principales problemas en la gestión del agua -la sequía, el principal de ellos, pero también la realidad adversa del cambio climático, las inundaciones recurrentes, el agotamiento de acuíferos, la contaminación, el servicio precario en redes de agua potable y alcantarillado, etc.- no han estado en la agenda de su consejo de cuenca. La agenda de este último se encuentra atorada en el tema administrativo, alineada al formalismo y a la necesidad de justificar un modelo de gestión participativo que sea útil para promover discur- 
sivamente la imagen del país ante los foros y organismos internacionales (Martínez, Martínez, y López, 2015).

Es cierto que hay actores que abanderan agendas alternativas. Los más emblemáticos, pero no los únicos, son el movimiento que pugna por el saneamiento del río Santiago en el corredor industrial de Jalisco (Martínez y Hernández, 2009) y el movimiento opositor a la presa de El Zapotillo (Ochoa, 2013), que incluso ya logró incorporar algunas de sus demandas a la agenda del Gobierno de Jalisco. Son este tipo de actores y de movimientos locales quienes tendrían la posibilidad de generar un cambio e incidir en los problemas del agua. Desafortunadamente, no participan directa o indirectamente en el consejo de cuenca para el caso del río Santiago, e incluso algunos de ellos han sido bloqueados sistemáticamente de formar parte del mismo.

Se debaten actualmente dos propuestas de reformas al marco normativo. Una es la que restringe la participación y abandona, en los hechos, el modelo GIRH para privilegiar intereses empresariales y restringir el concepto de gobernanza a la legalidad y la eficiencia (Murillo Licea, 2012). Esta es la apuesta que se hace en el marco de la Ley General de Aguas que en los últimos meses ha propuesto el Poder Legislativo (Romero, 2016). Otra alternativa es la llamada propuesta ciudadana (Agua para Todos, 2015), que busca introducir un modelo de plena participación social y una gobernanza del agua más cercana a lo estipulado dentro del modelo GIRH, construida de abajo hacia arriba, con una mejor representatividad y un diseño más incluyente (Murillo Licea, 2012).

Ahora bien, dado el caso de que en el Consejo de Cuenca del río Santiago privan reglas no formales caracterizadas por la apatía, la exclusión y la discrecionalidad (en detrimento de un marco normativo que promueve formalmente la coordinación, la participación y la gobernanza), resulta sensato pensar que las reglas informales seguirán influyendo 
en las elecciones estratégicas de los actores involucrados independientemente de la alternativa que se adopte para actualizar el marco normativo.

En consecuencia, se propone transitar hacia el reconocimiento de estas prácticas informales e incidir en ellas, sobre todo en espacios locales, para lograr una transformación que nos acerque a un gobierno del agua eficiente, participativo, sustentable y equitativo.

Actas Cocurs (2016). Actas de las sesiones del Consejo de Bibliografía Cuenca del río Santiago [actas sin clasificar en el Archivo de la Secretaría Técnica]. Guadalajara, México.

Agua para Todos (2015). Propuesta ciudadana para la Ley General de Aguas. Recuperado de: https://agua.org.mx/ wp-content/uploads/20 18/09/propuesta-ciudadana-leygeneral-de-aguas-febrero-20 I5.pdf

Aguilar, L. F. (1993). Problemas públicos y agenda de gobierno. México: Porrúa.

Auditoría Superior de la Federación (2015). Informe del resultado de la fiscalización superior de la cuenta pública 2013. Auditoría de desempeño: I3-0-I 6B00-07-0 / 43. Grupo Funcional Desarrollo Económico. Recuperado de: http:// www.asf.gob.mx/Trans/Informes/IR20 I3i/Documentos/ Auditorias/2013_0I43_a.pdf

Barkin, D.,y Klooster,D. (2006).“Estrategias de la gestión del agua urbana”, en D. Barkin (coord.), La gestión del agua urbana en México. Retos, debates y bienestar (pp. I0-2I). México: ANEAS, Universidad de Guadalajara.

Barreda, A. (2006). En defensa del agua. México: Sindicato Mexicano de Electricistas, Editorial Ítaca.

Becker, G. (1995). Human Capital and Poverty Alleviation. Working Papers $N^{\circ}$ 52. Washington: The World Bank. Recuperado de: http://documents.worldbank.org/ 
Bibliografía
curated/en/I 2 I79 | 468764735830/Human-capital-andpoverty-alleviation

Bravo, G. (2005). "Esquemas de participación comunitaria en la cuenca del río Grande/río Bravo”, en S. Vargas, y E. Mollard (eds.), Problemas socio-ambientales y experiencias organizativas en las cuencas de México (Pp. 356367). Ciudad de México: IMTA/ SEMARNAT. Recuperado de: https://agua.org.mx/wp-content/uploads/2007/06/ Problemas-Socio-Ambientales-y-Experiencias-Organizativas-en-las-Cuencas-de-Mexico.pdf

Cejudo, G. M. (2008). Discurso y políticas públicas: enfoque constructivista (Discourse and Public Policy: A Constructivist Approach). Documento de trabajo del CIDE, número 205. México: CIDE. Recuperado de: https://ssrn.com/ abstract $=2495550$

Cobb, R.W.,y Elder, C. (1974). Participation in American Politics: The Dynamics of Agenda-Building. Baltimore:Johns Hopkins University Press. doi: https://doi.org/ 10.2307// 958664

Comisión Nacional del Agua (2010). Documentos básicos de los consejos de cuenca. México: Comisión Nacional del Agua. Recuperado de: http://sgpwe.izt.uam.mx/files/ users/uami/patt/2._Conceptos_basicos/Documentos_basicos_de_los_consejos_de_cuenca.pdf

Comisión Nacional del Agua (20I5). Programa de medidas preventivas y de mitigación contra la sequía del Consejo de Cuenca del río Santiago. México: Comisión Nacional del Agua. Recuperado de: https://www.gob.mx/cms/uploads/ attachment/file/99956/PMPMS_CC_R_o_Santiago_R. pdf

Congreso de los Estados Unidos Mexicanos (2016). Ley de Aguas Nacionales. Recuperado de: http://www.diputados. gob.mx/LeyesBiblio/pdf/I6_240316.pdf

Crozier, M., y Friedberg, E. (1990). El actor y el sistema. Las restricciones de la acción colectiva. México:Alianza Editorial Mexicana. 
Flores Elizondo, R. (20I2). “Los Consejos de Cuenca en México como espacio de gobernanza. El caso de la cuenca Lerma-Chapala durante la sequía de 19972003”, en H. Ochoa-García, y H. J. Bürkner (coords.), Gobernanza y gestión del agua en el Occidente de México: la metrópoli de Guadalajara (pp. 73-100). Guadalajara: ITESO. Recuperado de: https://rei.iteso.mx/bitstream/ handle/ I I I 7/454/ConsejosDeCuenca-Gobernanza. pdf? sequence $=2$

Galindo Sosa, J. A. (2009). Indicadores clave de desempeño de los Consejos de Cuenca [tesis de maestría inédita]. Instituto Mexicano de Tecnología del Agua: México. Recuperado de: http://hdl.handle.net/20.500.12013/I084 Global Water Partnership (2009). Manual para la gestión integrada de recursos hídricos en cuencas. Francia: Global Water Partnership e International Network of Basin Organizations. Recuperado de: https://www.rioc.org/ IMG/pdf/RIOC_GWP_Manual_para_la_gestion_integrada.pdf

Global Water Partnership (20I3). Guía para la aplicación de la gestión integrada del recurso hídrico (GIRH) a nivel municipal.Tecucigalpa:GWP. Recuperado de: https://www. gwp.org/globalassets/global/gwp-cam_files/guia-girh-aescala-municipal.pdf

Grupo de Trabajo Buena Gobernanza (2012). Hacia una buena gobernanza para la gestión integrada de los recursos hídricos. Documento de posicionamiento de México en el vI Foro Mundial del Agua. Francia: GTBG. Recuperado de: http://www.oas.org/en/sedi/dsd/iwrm/past\%20events/ D7/6\%20WWF-GOBERNANZA\%20Final.pdf

Guber, R. (200I). La etnografia: método, campo y reflexividad. Buenos Aires: Grupo Editorial Norma.

Guzmán Arroyo, M. (200I). "Consejos y comisiones en la cuenca Lerma-Chapala”. Renglones, (49), 65-69. Recuperado de: http://hdl.handle.net/ I I I 7/440

Bibliografía 
Bibliografía
Instituto Nacional de Estadística y Geografía (1988). Red hidrográfica escala 1:50 000. Edición 2.0. México: Inegi. Recuperado de: http://www.inegi.org.mx/geo/contenidos/topografia/regiones_hidrograficas.aspx

Instituto Nacional de Estadística y Geografía (2015). Conteo de población y vivienda, 20I5. Recuperado de: http://www. inegi.org.mx/est/contenidos/proyectos/ccpv/cpv20I0/ Default.aspx

Long, N. (2007). Sociología del desarrollo: una perspectiva centrada en el actor. México: Centro de Investigaciones y Estudios Superiores en Antropología Social.

Maldonado, C., y Casar M. (2008). Formación de agenda y procesos de toma de decisiones: una aproximación desde la ciencia política. Documentos de trabajo CIDE 2008. Núm. 207. México: CIDE. Recuperado de: http://repositoriodigital.cide.edu/handle/II65I/8I5

Maldonado, C., Valera, A., y Palma, F. (20I3). La construcción de pactos y consensos en materia de política social: apuntes para un marco de análisis. Santiago de Chile: CEPAL. Recuperado de: https://repositorio.cepal.org/ handle/ I I362/6/96

Martínez, P.,y Hernández, E. (2009).“Impactos de la contaminación del río Santiago en el bienestar de los habitantes de El Salto,Jalisco". Espacio abierto, I 8(4), 709-729. Recuperado de:http://produccioncientificaluz.org/index.php/ espacio/article/view/ / 348// 350

Martínez,J., Martínez,J.,y López,J.(20I4).“Avances y restricciones en la gestión democrática del agua en México". Plurimondi, VII(I5), 5I-72. Recuperado de: http://plurimondi.poliba.it/index.php/Plurimondi/article/view/89

Merino, M., Arellano Gault, D., Cejudo, G. M., Sour, L., Santibáñez, L., Mariscal, J., y Bracho, T. (2010). Problemas, decisiones y soluciones: enfoques de política pública. México: Fondo de Cultura Económica. 
Murillo Licea, D. (coord.) (20I2). Gobernanza del agua, un Bibliografía desafio actual. Hacia una mirada crítica del concepto y de su aplicación. México: IMTA. Recuperado de: repositorio. imta.mx/bitstream/20.500. I 20 I3/ I528/ I/CP-I I I0. I.pdf Mussetta,P.(2009).“Participación y gobernanza. El modelo de gobierno del agua en México”. Espacios públicos, I2(25), 66-84. Recuperado de: http://www.redalyc.org/articulo. oa? id $=676$ I 1350005

Narváez, J. (2005). La persona en el derecho civil. México: Porrúa.

Ochoa, H. (20/3). “iAlternativas para la gestión del agua y el desarrollo regional? Conflicto por la presa El Zapotillo”. Observatorio del desarrollo. Investigación, reflexión y análisis, II(7), I6-2I. Recuperado de: https://rei.iteso.mx/ bitstream/handle/ I I I I 7/ |420/EOchoa-El\%20Zapototillo.pdf? sequence $=4$

Olavarría, M. (2007). Conceptos básicos en el análisis de politicas públicas. Documentos de trabajo. Instituto de Asuntos Públicos, Universidad de Chile. Chile:Universidad de Chile. Recuperado de: http://repositorio.uchile.cl/bitstream/ handle/2250/I 23548/Conceptos_\%20Basicos_Politicas_Publicas.pdf?sequence $=$ I

Palerm, J., y Rodríguez, B. (2005). "Espacios de negociación: autogestión y Estado en el río Cuautla", en S. Vargas, y E. Mollard (eds.), Problemas socio-ambientales y experiencias organizativas en las cuencas de México (PP. 172-193). Ciudad de México: IMTA/SEMARNAT. Recuperado de: http://horizon.documentation.ird.fr/exl-doc/ pleins_textes/divers I2-05/0 I0038079.pdf

Parra-Armenta, E., y Salazar A. (20I8). “La gestión integral del agua en dos consejos de cuenca del noroeste de México". Entreciencias. Diálogos en la sociedad del conocimiento, 6(17), 79-94. doi: http://dx.doi.org/I0.2220I/ enesl.20078064e.20I7.I5.62580 
Bibliografía
Pérez, E. (20/3a). Entrevista personal con Informante I. Guadalajara, Jalisco, México.

Pérez, E. (20I3b). Entrevista personal con Informante 2. Guadalajara, Jalisco, México.

Pérez, E. (20I5a). Entrevista personal con Informante 3. Guadalajara, Jalisco, México.

Pérez, E. (20I5b). Entrevista personal con Informante 4. Guadalajara, Jalisco, México.

Pérez, E. (20I5c). Entrevista personal con Informante 5. Guadalajara, Jalisco, México.

Pérez, E. (20I5d). Entrevista personal con Informante 6 . Guadalajara, Jalisco, México.

Revuelta, B. (2007). “La implementación de políticas públicas”. Díkaion, 2 I(I6), I35-I56. Recuperado de: http:// dikaion.unisabana.edu.co/index.php/dikaion/article/ view/I379/I5 I5

Romero, L. (20I6). "Participation and Legislation Involving Water in Mexico: A Historical Approach”. Agua y territorio, (7), 22-34. doi: https://dx.doi.org/ I0.1756 I/at.v0i7

Ruiz, R. (2015). "Policy convergence to Integrated Water. Resources Management in Mexico". Revista mexicana de análisis político y administración pública, IV(2), 67-88. Recuperado de: http://www.remap.ugto.mx/index.php/ remap/article/download// 32/I I4

Sánchez, E. (2010). Enfoque multidimensional de la gestión integrada de recursos naturales en la cuenca del río San Pedro [tesis inédita de doctorado]. Instituto Mexicano de Tecnología del Agua: México. Recuperado de: http:// repositorio.imta.mx/handle/20.500.12013/343

Sancho, T., y Parrado, S. (2004). “Los organismos de cuenca de España y los consejos de cuenca mexicanos, análisis comparativo y reflexiones". Revista de obras públicas, (3444), I7-34. Recuperado de: http://ropdigital.ciccp.es/ pdf/publico/2004/2004_mayo_3444_02.pdf 
Santander, P. (20II). "Por qué y cómo hacer análisis de discurso". Cinta moebio, (4I), 207-224. Recuperado de: www.moebio.uchile.cl/4l/santander.html

Secretaría de Hacienda y Crédito Público (20 I6). Datos del programa U0I5 Programa de desarrollo organizacional de los Consejos de Cuenca. Recuperado de: https://www. sistemas.hacienda.gob.mx/ptpsed/datosProgramaLlave. do? id $=16 \mathrm{U} 015$

Soares, D., y Murillo, D. (20I3). “Gestión de riesgo de desastres, género y cambio climático. Percepciones sociales en Yucatán, México". Cuadernos de desarrollo rural, I0(72), I8I-199. Recuperado de: http://revistas.javeriana.edu. co/index.php/desarrolloRural/article/view/7029/5588

Soares, D., y Vargas, S. (2008). La gestión de los recursos hídricos: realidades y perspectivas. Tomo I. Jiutepec: IMTA, U. de G. Recuperado de: http://repositorio.imta.mx/ handle/20.500.120I3/II60

Vargas, S., y Mollard E. (eds.) (2005). Problemas socio-ambientales y experiencias organizativas en las cuencas de México. Ciudad de México: IMTA/semarnat. Recuperado de: http://horizon.documentation.ird.fr/exl-doc/pleins_ textes/divers I 2-05/0 10038079.pdf 